\title{
B. $\operatorname{Rey}^{1}$ \\ U. Jacob ${ }^{2}$ \\ Modalités d'exploitation des caprins dans la province de Ngozi, Burundi
}

REY (B.), JACOB (U.).Modalités d'cxploitation des caprins dans la province de Ngozi, Burundi. Revue Élev. Méd. vét. Pays trop., 1993, 46 (4) : $637-644$

Cet article présente les modalités d'exploitation des caprins dans la province de Ngozi au Burundi. Les données ont été recueillies sur deux ans par un suivi zootechnique de 251 éleveurs. Parmi ceux-ci, 158 avaient des caprins croisé Alpine et étaient encadrés par le Projet caprin de Ngozi. L'évolution de la taille et de la composition génétique des troupeaux d'une part, les pratiques de vente, d'autoconsommation et de traite d'autre part, sont analysées. Les variations de comportement des différents types d'éleveurs sont soulignées. La fonction d'épargne que jouent les caprins dans ce système agraire a eu comme résultat une forte fluctuation de cette population au sein des troupeaux et la permutation des éleveurs avec l'abandon de l'élevage caprin par 6,3 p. 100 d'entre eux chaque année. Ce risque d'abandon est d'autant plus marqué que le troupeau est de petite taille, tout en restant indépendant de la possession de caprins croisés. L'adoption des caprins croisés par une fraction des éleveurs s'est accompagnée de modifications des pratiques d'exploitation. Soixante-cing p. 100 d'entre eux ont accepté la traite et la vente du lait ; ils ont vendu leurs chèvres à un âge moyen plus élevé, alors que les chevreaux l'ont été au même âge. Les chevreaux les plus lourds de la race locale ont néanmoins fait l'objet d'une sélection, au contraire des chevreaux croisés. Les performances numériques d'exploitation ont cependant été inférieures chez ces éleveurs, qui ont par ailleurs maintenu dans leurs troupeaux une fraction constante de caprins de race locale.

Mots clés : Caprin - Chèvre Alpine - Système d'élevage - Croisement Amélioration des animaux - Production laitière - Commercialisation Économie de l'élevage - Rurundi.

\section{INTRODUCTION}

Les hautes terres d'Afrique centrale (Burundi, Rwanda, est du Zaïre, Ouest tanzanien et Ouganda) sont caractérisées par de fortes densités de population rurale. Les systèmes agraires, fondamentalement agricoles, évoluent rapidement pour s'adapter à une pression foncière croissante. Dans le domaine agricole, on observe une intensification de l'utilisation du milieu : réduction des jachères, association de cultures, mise en culture de terres marginales. Dans le domaine de l'élevage, la population bovine régresse progressivement au bénéfice des petites espèces (ovins, caprins, volailles, rongeurs) qui consomment moins d'espace et valorisent mieux la main-d'œuvre excédentaire. Ces espèces ont fait l'objet, au cours de la dernière décennie, d'un nombre croissant

\footnotetext{
1. CIRAD-EMVT, mis à la disposition du CIPEA, POB 5689, AddisAbeba, Éthiopie.

2. GTZ/Projet caprin de Ngozi, BP 45, Ngozi, Burundi.

Reçu le 21.7.1993, accepté le 5.10.1993
}

de projets de développement. Lorsqu'ils concernent les caprins, ils sont souvent fondés sur un programme de croisement avec des génotypes exotiques, utilisant soit des races à aptitude viande (Boer), soit des races laitières (Alpine, Toggenburg par exemple).

Le projet caprin de Ngozi au Burundi fonctionne depuis 1980 dans le cadre de la coopération entre le ministère de l'Agriculture et de l'Elevage du Burundi et le ministère de la Coopération économique allemand. Son objectif est une augmentation des revenus des petites exploitations à travers l'amélioration de l'élevage caprin et la promotion de sa production laitière (7). L'activité principale est le croisement des chèvres de race locale avec des boucs de race alpine mis à la disposition des éleveurs dans des centres de monte. Le croisement avec du matériel génétique introduit devient la principale innovation technologique autour de laquelle est organisée la vulgarisation de cultures fourragères, de traitements vétérinaires et la construction d'une étable. L'achat du lait et sa commercialisation sont assurés par le projet dans un rayon de 20 $\mathrm{km}$ environ. En 1989, l'encadrement individuel de tous les éleveurs croisant au moins une de leurs chèvres dans un centre de monte a été interrompu au profit d'un encadrement axé sur la création de groupements d'éleveurs.

Ce projet vise donc à modifier les modalités d'élevage autour de l'acquisition de caprins croisés. S'appuyant sur l'analyse des données collectées durant deux ans par un suivi conjoint du Centre international pour l'Elevage en Afrique (CIPEA/ILCA) et du projet caprin de Ngozi, cet article compare les pratiques d'exploitation des caprins de race locale et croisés chez différents types d'éleveur. II étudie ainsi les modifications induites par le projet, et en particulier celles relatives à l'exploitation "viande". La compréhension des stratégies sous-jacentes permet d'envisager les conditions nécessaires à la pérennité de tels projets de croisement pour de petits troupeaux caprins dans des systèmes à dominante agricole.

L'évolution de la taille et du croît de ces troupeaux, ainsi que celle de leur composition génétique, reflète les comportements paysans quant à leur gestion. Leur exploitation sera considérée en analysant les pratiques de vente, d'autoconsommation et de traite.

L'altitude moyenne de la province de Ngozi (nord du Burundi) est de $1800 \mathrm{~m}$ et la pluviométrie annuelle d'environ $1400 \mathrm{~mm}$, avec un régime saisonnier bimodal. Les températures annuelles varient entre $6,6^{\circ} \mathrm{C}$ et $28,2^{\circ} \mathrm{C}$. La plupart des activités économiques se rapportent à l'agriculture qui emploie 97 p. 100 de la population (495 000 habitants en 1985) (8). 
Le système agricole repose sur les cultures vivrières suivantes : haricots, bananes, maïs, manioc, patate douce, colocase et sorgho. Le café est la principale culture de rente. Depuis quelques années la pression démographique (337 habitants par $\left.\mathrm{km}^{2}\right)$ a conduit à l'aménagement et à la mise en culture des bas-fonds en saison sèche, pour le riz essentiellement. Ces activités répartissent les besoins spécifiques en main-d'œuvre tout au long de l'année, avec un pic d'emploi lors de la période de récolte du café, de mars à juin (5).

\section{MATÉRIEL ET MÉTHODES}

Un échantillon de troupeaux caprins, répartis dans les sept communes qui constituent le périmètre laitier du projet, a fait l'objet d'un suivi démographique et de productivité entre novembre 1989 et décembre 1991. Cent quatre exploitations ont été choisies de façon aléatoire parmi celles encadrées par le projet après avoir participé au programme de croisement, auxquelles s'ajoutent 93 exploitations pratiquant un élevage "traditionnel", sans chèvres croisées. Par ailleurs, 54 éleveurs ont été choisis par le projet comme de futurs producteurs d'animaux améliorés en milieu rural. Ces "sélectionneurs", font l'objet d'un encadrement particulier mais sont restés soumis au même suivi. Les auteurs (6) ont montré qu'il n'y avait pas de différence de structure d'exploitation entre les élevages traditionnels et encadrés. Les éleveurs sélectionneurs disposent par contre de plus de terre et de force de travail familiale.

Le suivi des caprins individuellement identifiés s'est fait à raison de deux visites par mois. II a été précédé d'une enquête retrospective sur les structures d'exploitation et les pratiques d'élevage et a concerné un effectif moyen de 1600 animaux. Quarante-cinq p. 100 de ces caprins étaient de race locale ; 19 p. 100 demi-sang Alpine (F1) et 22 p. 100 trois-quarts Alpine (R1). Les 14 p. 100 restant correspondent aux autres niveaux de croisement. Les événements démographiques, naissances, achats, ventes, etc., et la quantité de lait trait ont été enregistrés à chaque visite. Les chevreaux ont été pesés une fois par mois, et les adultes chaque trimestre.

L'abandon de tout élevage caprin durant la période suivie a été analysé avec un modèle de régression logistique utilisant les 3 facteurs suivants : type d'élevage, commune, nombre de chèvres adultes au début du suivi.

L'âge à la vente et l'âge à l'abattage familial ont été analysés en utilisant un modèle linéaire comprenant 5 facteurs : sexe et génotype, type d'élevage et interactions (sexe $x$ génotype) et (sexe $x$ type d'élevage).

Les critères de vente des chevreaux mâles ont été étudiés en analysant leur probabilité d'être toujours présents dans le troupeau (vs être vendus) à l'âge d'un an, à l'aide d'un modèle de régression logistique utilisant leur poids à 180 jours et leur saison de naissance (sèche/pluies). Le modèle a utilisé pour les chevreaux croisés une fonction directe du poids vif ; celui pour les chevreaux de race locale est une fonction quadratique.

\section{RÉSULTATS}

\section{Évolution de la population suivie}

\section{Nombre d'éleveurs}

Entre janvier 1990 et décembre 1991, 25 des 251 exploitants suivis ont abandonné tout élevage caprin, soit 13 élevages traditionnels, 11 encadrés et 1 éleveur sélectionneur, soit respectivement 14,11 et 2 p. 100.

Parmi les causes de ces abandons, les raisons volontaires dominent. Treize éleveurs ont vendu toutes leurs chèvres, la plupart pour satisfaire des besoins monétaires immédiats tels que : frais scolaires, mariages etc. Quatre en ont fait don à leurs héritiers. Un de ces éleveurs s'est débarassé de toutes ses chèvres pour acheter un bovin pour des raisons de prestige. Parmi les autres raisons, cinq ont abandonné l'élevage à la suite d'une forte mortalité et deux après le vol de leur cheptel.

Par contre, les éleveurs-sélectionneurs, soumis à un encadrement plus rapproché, et dotés des meilleures structures d'exploitation (6) n'ont pas abandonné. La régression logistique sur le risque d'abandon pour les élevages traditionnels et encadrés n'a pas démontré d'effet du type d'élevage. L'abandon de l'élevage caprin a donc été indépendant de la possession ou non de chèvres croisées. La taille du troupeau au début du suivi, exprimée par le nombre de femelles adultes, ajusté pour la commune enquêtée, a par contre eu un effet significatif $(\mathrm{P}<0,01)$. La figure 1 présente la probabilité d'abandon en deux ans de l'élevage caprin en fonction du nombre initial de chèvres : celle-ci a été de 5,38 p. $100\left(s_{m}=\right.$ 2,150 ) avec un troupeau de 3 chèvres et de 0,71 p. 100 $\left(s_{m}=0,689\right)$ avec un troupeau de 5 chèvres. Enfin, le risque d'abandon a varié suivant les communes, enquêtées $(P<0,01)$

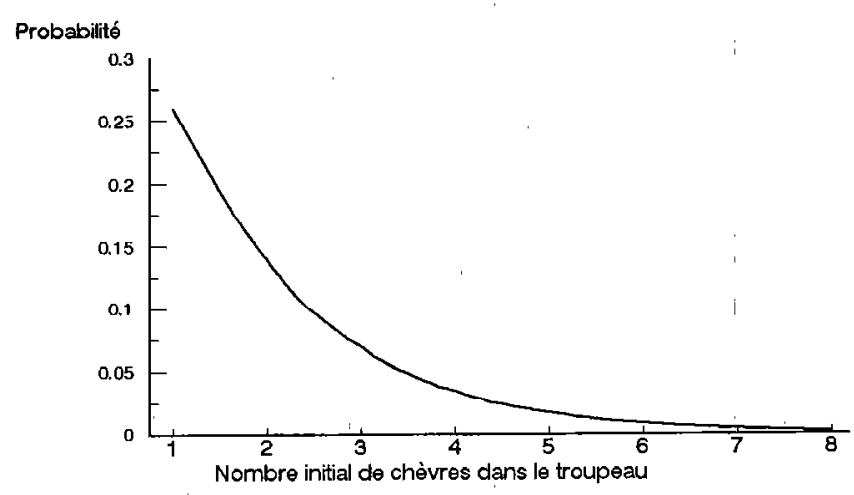

Figure 1 : Probabilité de disparition d'un élevage caprin en deux ans en fonction du nombre initial de chèvres dans le troupeau. 


\section{Croît du troupeau}

Les troupeaux caprins dans la région de Ngozi sont de petite taille : 5,8 caprins dont 2,8 chèvres adultes en moyenne. Aux fluctuations saisonnières près, le nombre de caprins présents chez chaque éleveur traditionnel ayant conservé des animaux est resté stable entre décembre 1989 et décembre 1991. En revanche, le nombre de caprins par élevage a diminué dans les deux autres catégories (tableau I).

Le croît numérique brut des troupeaux (effectif de fin d'année moins effectif de début d'année divisé par l'effectif de début d'année) a varié d'une année à l'autre: $+6,7$ p. $100\left(S_{m}=3,7\right)$ en 1990 et $-7,4$ p. $100\left(S_{m} 3,8\right)$ en 1991 $(P<0,01)^{m}$. L'effet du type d'élevage sur le croit brut des troupeaux en 1990 et en 1991 considérés indépendamment n'a cependant pas été significatif.

\section{Composition génétique des troupeaux}

Au début du suivi, tous les élevages traditionnels, à l'exception de l'un d'entre eux, ne possédaient que des animaux de race locale. En deux ans, trois autres éleveurs traditionnels ont obtenu au moins un animal croisé. L'évolution de la composition génétique des troupeaux suivis chez les éleveurs encadrés et chez les sélectionneurs est présentée dans la figure 2. Elle permet de remarquer les faits suivants:

- ces éleveurs ont conservé une fraction à peu près constante (de 24 à 21 p. 100) de leur cheptel en race locale sur les deux ans. Cette fraction a varié de 32 à 29 p. 100 dans les élevages encadrés et de 11 à 9 p. 100 chez les sélectionneurs. Quatre-vingt trois p. 100 des caprins de la race locale chez ces éleveurs étaient des chèvres ou des chevrettes ;

- la structure de la population de chèvres croisées s'est par contre modifiée notablement. Le pourcentage de croisés $\mathrm{F} 1$ a connu la plus forte baisse (de 33 à 22 p. 100 de la population suivie dans ces élevages), alors que celui des niveaux $\mathrm{R} 1$ et $\mathrm{R} 2$ est resté relativement constant avec 32 à 30 p. 100 , et 8 à 9 p. 100 . Cette diminution s'est effectuée au bénéfice des rétrocroisements avec des races locales et des autres croisements, dont la proportion a augmenté de 2 à 14 p. 100 .

\section{Exploitation et pertes}

Les figures 3 et 4 indiquent en nombre la mortalité et l'exploitation des cohortes de chevreaux et chevrettes nés en 1990 en fonction de leur âge jusqu'à un an. L'exploitation propre (ventes, abattage pour l'autoconsommation et dons) a été du même ordre de grandeur que la mortalité caprine pour l'ensemble des troupeaux : 1175 caprins morts et 1298 exploités sur deux ans. La vente des chevreaux a débuté dès l'âge de six mois. A un an, la moitié des chevreaux mâles ayant survécu avaient quitté l'exploitation, alors que ce pourcentage était plus faible pour les femelles (5 p.100).

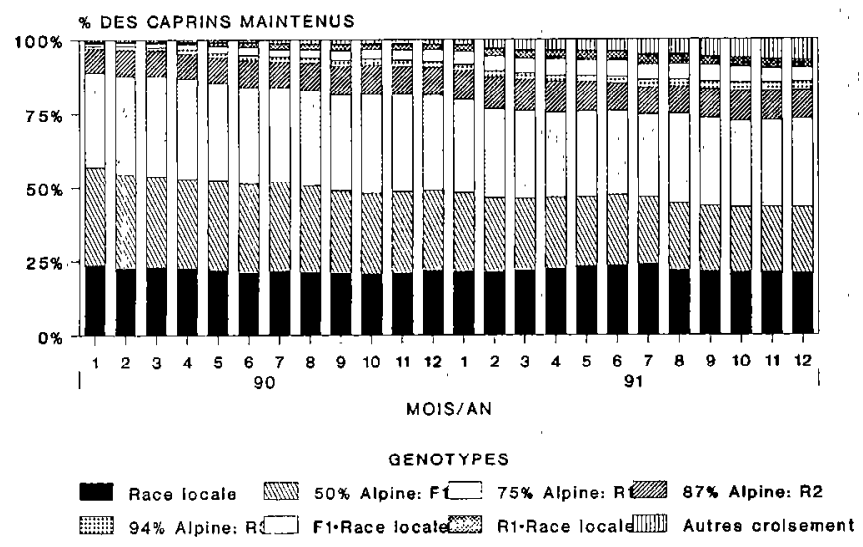

Figure 2 : Evolution de la composition génétique des troupeaux des élevages encadrés et sélectionneurs.

TABLEAU I Evolution de la taille moyenne des troupeaux caprins par type d'élevage au cours du suivi (en nombre de caprins par troupeau).

\begin{tabular}{|l|c|c|c|}
\hline Date & $\begin{array}{c}\text { Elevages } \\
\text { traditionnels }\end{array}$ & $\begin{array}{c}\text { Elevages } \\
\text { encadrés }\end{array}$ & $\begin{array}{c}\text { Elevages } \\
\text { sélectionneurs }\end{array}$ \\
\hline Décembre 1989 & 5,0 & 6,6 & 8,0 \\
Juin 1990 & 6,1 & 7,5 & 9,4 \\
Décembre 1990 & 5,5 & 6,4 & 8,4 \\
Juin 1991 & 6,0 & 6,5 & 8,4 \\
Décembre 1991 & 5,3 & 5,8 & 7,2 \\
\hline
\end{tabular}

\section{Ventes}

Les ventes ont concerné toutes les catégories d'animaux et les différents types d'élevages. Les animaux vendus ont surtout été destinés à l'abattage, parfois à l'élevage dans d'autres exploitations. Le pourcentage des animaux vendus parmi les populations des différents types génétiques a été identique, à l'exception des croisés R1 (75 p. 100 Alpine) qui ont fait l'objet de plus nombreuses transactions avec 282 ventes observées en deux ans pour un effectif mensuel moyen de 392 têtes.

Bien que des ventes aient lieu toute l'année, elles ont été plus nombreuses de juillet à octobre et de février à mars (figurc 5). Ces deux périodes correspondent aux époques d'achat des intrants pour les deux principales saisons de culture et sont directement liées aux besoins monétaires(5). II est remarquable à ce sujet que les vols de caprins aient également été enregistrés aux mois de février, mars et septembre.

L'analyse de variance de l'âge de 'vente des caprins a montré un effet significatif de leur sexe et de leur génotype, ainsi que du type d'élevage (tableau II). Les moyennes ajustées montrent que les chevreaux mâles ont été vendus à un an, sans qu'il y ait de différence entre les types génétiques ou entre les types d'élevages. L'âge de vente des femelles a par contre varié avec ces deux facteurs. Les chevrettes croisées ont été vendues plus jeunes que celles de race locale : 2 ans contre 3 ans 
B. Rey U. Jacob

TABLEAU II Âge à la vente et à l'abattage pour l'autoconsommation (moyennes établies par la méthode des moindres carrés).

\begin{tabular}{|c|c|c|c|c|c|c|c|}
\hline \multirow[b]{2}{*}{ Facteurs } & \multirow[b]{2}{*}{ Modalités } & \multicolumn{3}{|c|}{ Âge à la vente (j) } & \multicolumn{3}{|c|}{ Âge à l'abattage (j) } \\
\hline & & $n$ & $x$ & $s_{m}$ & $n$ & $x$ & $s_{m}$ \\
\hline $\begin{array}{l}\text { Moyenne } \\
\text { générale }\end{array}$ & (Prob.) & 1065 & $\begin{array}{c}0,0001 \\
701\end{array}$ & 15,8 & 108 & $\begin{array}{c}0,0001 \\
748\end{array}$ & 46,5 \\
\hline Sexe & $\begin{array}{l}\text { (Prob.) } \\
\text { Mâle } \\
\text { Femelle }\end{array}$ & $\begin{array}{l}554 \\
511\end{array}$ & $\begin{array}{c}0,0001 \\
369 \\
1033\end{array}$ & $\begin{array}{l}22,3 \\
22,2\end{array}$ & $\begin{array}{l}76 \\
32\end{array}$ & $\begin{array}{c}0,0001 \\
387 \\
1109\end{array}$ & $\begin{array}{l}50,9 \\
77,9\end{array}$ \\
\hline Génotype & $\begin{array}{l}\text { (Prob.) } \\
\text { Croisé } \\
\text { Race locale }\end{array}$ & $\begin{array}{l}593 \\
472\end{array}$ & $\begin{array}{c}0,0001 \\
541 \\
861\end{array}$ & $\begin{array}{l}26,4 \\
31,3\end{array}$ & $\begin{array}{l}60 \\
48\end{array}$ & $\begin{array}{c}0,0176 \\
585 \\
912\end{array}$ & $\begin{array}{l}78,0 \\
86,1\end{array}$ \\
\hline Type d'élevage & $\begin{array}{l}\text { (Prob.) } \\
\text { Traditionnel } \\
\text { Encadré } \\
\text { Sélectionneur }\end{array}$ & $\begin{array}{l}345 \\
420 \\
300\end{array}$ & $\begin{array}{c}0,0001 \\
533 \\
723 \\
845\end{array}$ & $\begin{array}{l}35,6 \\
27,7 \\
34,7\end{array}$ & $\begin{array}{l}35 \\
39 \\
34\end{array}$ & $\begin{array}{c}\mathrm{ns} \\
652 \\
855 \\
737\end{array}$ & $\begin{array}{r}112,3 \\
79,5 \\
92,1\end{array}$ \\
\hline $\begin{array}{l}\text { Sexe }(M / F)^{\star} \\
\text { génotype }\end{array}$ & $\begin{array}{l}\text { (Prob.) } \\
\mathrm{F}^{\star} \text { croisé } \\
\mathrm{F}^{\star} \text {. locale } \\
\mathrm{M}^{\star} \text { croisé } \\
\mathrm{M}^{*} \text { locale }\end{array}$ & $\begin{array}{l}259 \\
252 \\
334 \\
220\end{array}$ & $\begin{array}{c}0,0001 \\
731 \\
1335 \\
351 \\
387\end{array}$ & $\begin{array}{l}37,4 \\
39,0 \\
37,1 \\
49,1\end{array}$ & $\begin{array}{l}19 \\
13 \\
41 \\
35\end{array}$ & $\begin{array}{c}0,0430 \\
807 \\
1412 \\
362 \\
412\end{array}$ & $\begin{array}{r}127,3 \\
128,7 \\
90,3 \\
114,3\end{array}$ \\
\hline $\begin{array}{l}\text { Sexe }(M / F)^{\star} \\
\text { type d'élevage }\end{array}$ & $\begin{array}{l}\text { (Prob.) } \\
\mathrm{F}^{\star} \text { encadré } \\
\mathrm{F}^{*} \text { sélectionneur } \\
\mathrm{F}^{*} \text { traditionnel } \\
\mathrm{M}^{*} \text { encadré } \\
\mathrm{M}^{\star} \text { sélectionneur } \\
\mathrm{M}^{*} \text { traditionnel }\end{array}$ & $\begin{array}{l}206 \\
134 \\
171 \\
214 \\
166 \\
174\end{array}$ & $\begin{array}{c}0,0001 \\
1063 \\
1310 \\
725 \\
383 \\
380 \\
342\end{array}$ & $\begin{array}{l}37,0 \\
47,2 \\
48,6 \\
41,2 \\
50,9 \\
52,1\end{array}$ & $\begin{array}{r}12 \\
13 \\
7 \\
27 \\
21 \\
28\end{array}$ & $\begin{array}{l}\text { ns } \\
1345 \\
1078 \\
905 \\
365 \\
397 \\
399\end{array}$ & $\begin{array}{r}127,1 \\
137,1 \\
189,8 \\
95,6 \\
122,9 \\
120,1\end{array}$ \\
\hline
\end{tabular}

$n$ : nombre d'observation (pour chaque modalité) ; $x$ : moyenne estimée (méthode des moindres carrés); $S_{m}$ : erreur standard de l'estimation ; Prob. : niveau de signification statistique de l'effet considéré.

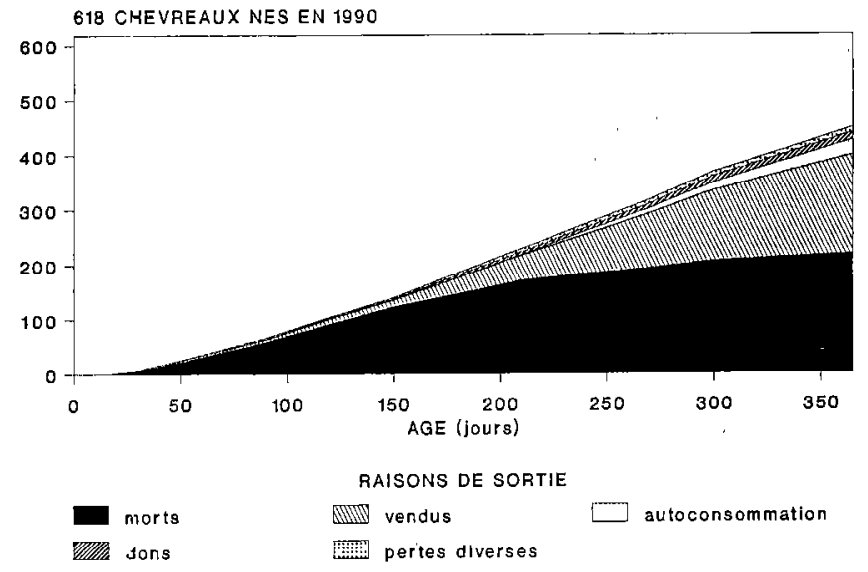

Figure 3 : Mortalité et exploitation des chevreaux nés en 1990.

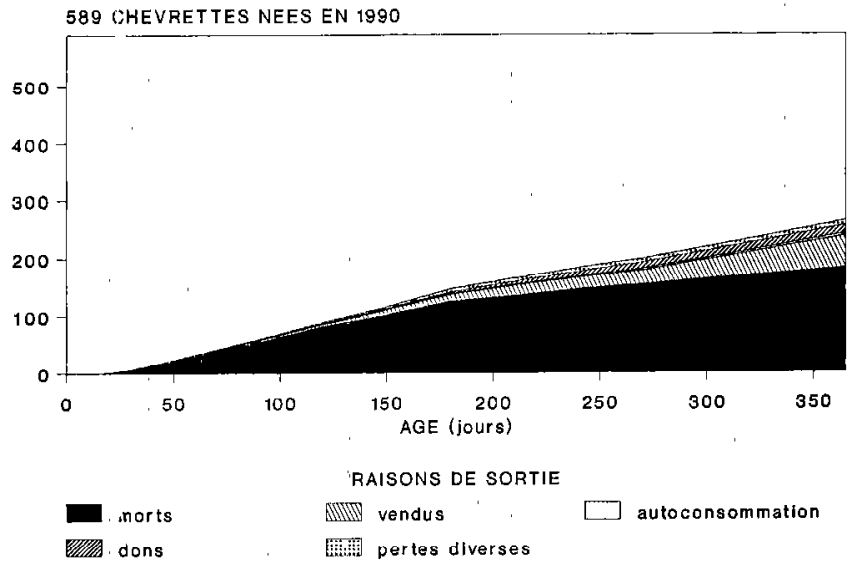

Figure 4: Mortalité et exploitation des chevrettes nées en 1990. 


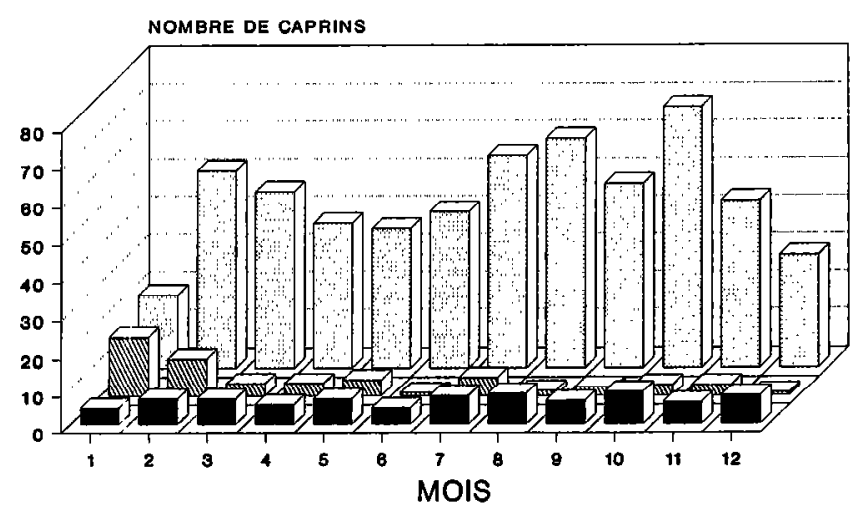

achat abat tage familial [D Ventes Sulvi de 251 exploitations

Figure 5 : Répartition mensuelle des ventes, achats et abattages familiaux (1990-1991).

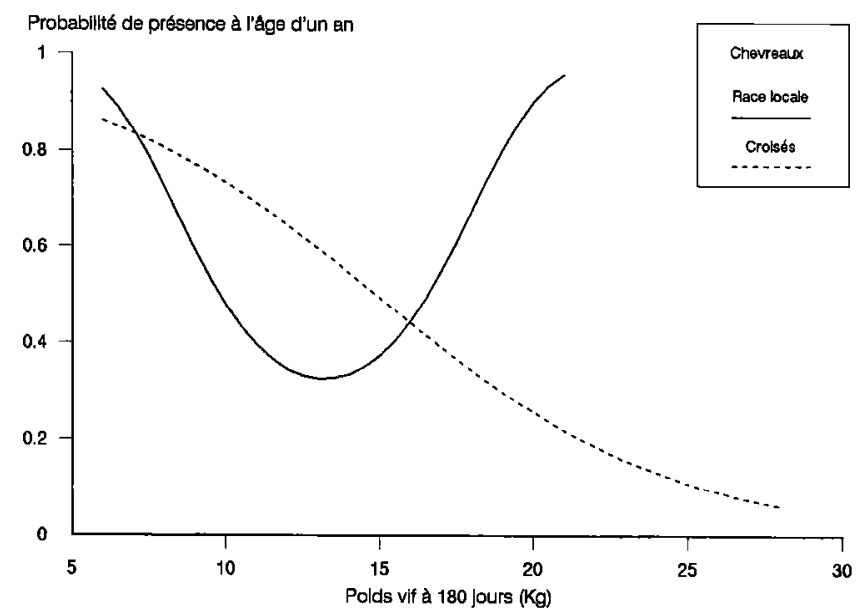

Figure 6 : Probabilité de la présence dans le troupeau d'un chevreau à un an en fonction de son poids vif à 180 jours.

et demi $(P<0,01)$. Elles ont été vendues à 2 ans dans les élevages traditionnels, à 3 ans par les éleveurs encadrés et à 3 ans et demi par les éleveurs sélectionneurs $(P<0,01)$.

Le modèle de regression logistique a été appliqué à un échantillon de 127 chevreaux de race locale et 188 chevreaux croisés pour lesquels le poids vif à six mois était connu, et qui ont été vendus entre 6 et 12 mois ou étaient encore présents à l'âge d'un an. Les facteurs suivants ont eu un effet significatif : la saison de naissance, le poids vif pour les deux génotypes, et le poids vif au carré pour les chevreaux de race locale uniquement.

La figure 6 présente la probabilité pour un chevreau vivant à 180 jours de ne pas être vendu avant l'âge d'un an en fonction de son poids à cet âge. Elle permet de constater ce qui suit :

- les chevreaux croisés ont eu d'autant plus de chance d'être vendus qu'ils étaient les plus lourds à 6 mois ; les animaux ayant eu la meilleure croissance ont donc quitté les élevages le plus tôt ;
- la situation des chevreaux de race locale a été différente : le poids moyen à six mois des chevreaux vendus n'était pas différent de celui des chevreaux conservés. Jusqu'à $12 \mathrm{~kg}$ de poids vif, la tendance est similaire à celle observée pour les chevreaux croisés. Les individus les moins performants ont été conservés pour être vendus après l'âge d'un an. Au-delà du poids vif à 6 mois, la tendance est inversée et les chevreaux de race locale les plus lourds ont été conservés.

\section{Autoconsommation et dons}

Le nombre d'animaux abattus pour l'autoconsommation a été faible : 108 , soit $6 p$. 100 de l'effectif mensuel moyen suivi. L'abattage familial a touché en plus grande proportion les animaux croisés avec 60 contre 48 de race locale. Un tiers des animaux abattus étaient des femelles. L'autoconsommation est apparue concentrée à l'époque du Nouvel An, et dans une moindre mesure en juillet (figure 5), mois durant lequel la situation financière des exploitations est meilleure après la récolte du café, et pendant lequel les mariages sont plus nombreux (2).

Un faible pourcentage des éleveurs a pratiqué l'abattage familial. Cent huit têtes ont été abattues chez 72 éleveurs : 39 caprins chez 23 encadrés, 34 chez 21 sélectionneurs et 35 chez 28 éleveurs traditionnels. L'abattage familial a donc plutôt concerné les sélectionneurs.

L'âge moyen à l'abattage a varié avec le sexe et le génotype des chevreaux et a pris des valeurs similaires à celles de l'âge à la vente (tableau II). Contrairement à ce dernier, il n'a cependant pas varié selon le type d'élevage, quel que soit le sexe des chevreaux considérés.

Cent neuf animaux ont fait l'objet de dons, les mâles en moyenne à, l'âge de dix mois, les femelles vers 18 mois. II n'y a pas eu de différence selon les types génétiques ou les types d'élevage.

\section{Taux d'exploitation}

Le taux d'exploitation annuel, défini comme le rapport du nombre d'animaux vendus, abattus ou donnés au nombre de caprins présents en début d'année, a été différent $(P<0,05)$ en 1990 et $1991: 39$ p. $100\left(S_{m}=2,4\right)$ et 47 p. $100\left(S_{m}=2,422\right)$ respectivement. Bien qu'aucune différence ne soit détectée entre les types d'élevage pour chaque année prise indépendamment, le taux moyen sur deux ans a présenté une différence significative $(P<0,01)$ entre les élevages traditionnels d'une part ( 49 p. $100, S_{m}$ $=2,9$ ) et les élevages encadrés et sélectionneurs de l'autre (respectivement 39 p. $100 \mathrm{~S}_{\mathrm{m}}=2,7$ et 40 p. $100 \mathrm{~S}_{\mathrm{m}}$ $=3,6)$.

\section{Achats}

Les éleveurs concernés ont acheté peu de caprins en 1990 et 1991 : 163 têtes. Les achats ont surtout concerné des femelles (129/163), dans une proportion compa- 
rable à celle des femelles présentes dans l'échantillon suivi. Sur le plan génétique, les caprins de race locale étaient dominants $(95 / 163)$, et peu d'animaux de niveaux de croisement élevé (supérieur à $3 / 4$ alpine) ont été achetés.

\section{Traite des chèvres croisées}

Les chèvres de race locale ne sont pas traites au Burundi. La logique sous jacente au croisement des chèvres avec des géniteurs de race Alpine résidait dans la possibilité de les traire pour améliorer l'alimentation humaine (7). La figure 7 indique, sur la base des mesures effectuées par les enquêteurs, la fraction des chèvres croisées traites par les éleveurs dans les semaines suivant la mise bas. Le lait était destiné à la vente soit au projet, soit sur un marché local (où il se substitue au lait de vache), soit à l'autoconsommation.

Durant les 3 premiers mois de lactation, près de la moitié des chèvres croisées ont été traites le jour de la visite de l'enquêteur. Ce pourcentage a été supérieur pour les chèvres de niveau élevé de croisement. II a ensuite dimi. nué progressivement.

Le comportement des éleveurs sélectionneurs vis-à-vis de la pratique de la traite a été différent de celui des éleveurs encadrés. Sur deux ans, la presque totalité des premiers ont trait au moins une fois devant l'enquêteur, alors que ce pourcentage n'était que de 65 p. 100 chez les seconds. De même, chaque mois, une moyenne de 60 p. 100 des sélectionneurs et de 30 p. 100 des éleveurs encadrés ont trait leurs chèvres.

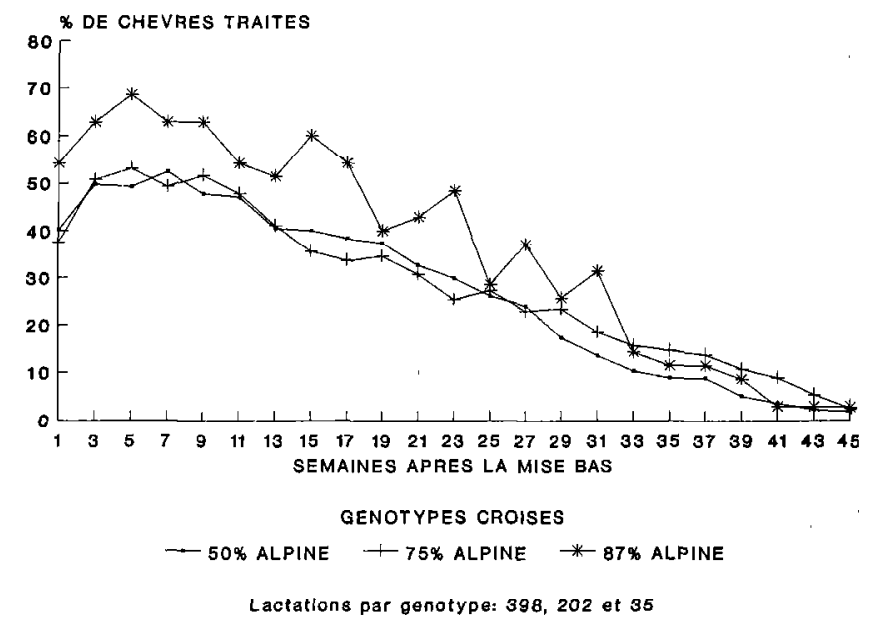

Figure 7 : Pourcentage de chèvres croisées traites dans les semaines suivant la mise bas.

\section{DISCUSSION}

\section{Les conséquences du rôle des caprins dans ces systèmes de production}

Les pratiques d'exploitation observées lors de ce suivi d'élevages confirment le rôle de réserve monétaire facilement mobilisable attribué aux caprins par la primauté donnée à laivente sur l'autoconsommation, l'époque et les types d'animaux vendus. La plupart de ces ventes correspondent à des abattages effectués par des bouchers en milieu urbain, et non à des transactions entre éleveurs. Le nombre limité d'animaux achetés par les éleveurs suivis, et l'évolution du croît des troupeaux reflètent par ailleurs la faible capacité d'épargne de ces systèmes de production. Des observations similaires sur le rôle microéconomique des petits ruminants ont été réalisées par FAUGÈRE et al. $(3,4)$ au Sénégal.

Le suivi de ces élevages a permis en outre d'apprécier les fluctuations de la possession de caprins en fonction de leur rôle économique. Si l'on exclut les sélectionneurs soumis à une plus grande pression de vulgarisation, le pourcentage de paysans abandonnant l'élevage est de 6,3 p. 100 par an, qu'ils aient ou non des animaux croisés. Il est cependant probable que dans le même temps, des paysans non suivis aient repris ou commencé l'élevage caprin comme forme de capitalisation. Le grand nombre de femelles vendues et la proportion des chèvres parmi les animaux achetés permettent de faire cette hypothèse. La relative constance du nombre de chèvres dans la province tient sans doute à ces permutations internes.

Cette pratique a plusieurs conséquences pour un projet de développement basé sur l'amélioration génétique. Elle réduit potentiellement son impact, puisque cette dernière est freinée par l'instabilité des élevages. Par ailleurs, la perception par les éleveurs du risque d'abandonner l'élevage peut ne pas les inciter aux investissements en travail et en capital exigés en contrepartie des génotypes introduits.

L'influence du nombre de femelles dans le troupeau sur le risque d'abandon étant notable, on pourrait penser à moduler le message de la vulgarisation sur ce critère : pour les petits troupeaux, viser à y' stabiliser l'élevage, en proposant éventuellement une source alternative de financement pour éviter la vente de caprins ; pour les plus grands, rechercher des investissements productifs.

\section{Les pratiques d'exploitation}

L'innovation technologique principale du projet est la modification des génotypes. L'ensemble des autres techniques proposées constitue le "produit technique" d'accompagnement tels que logement, traitements sani- 
taires, etc. La gestion de ce matériel génétique était la première pratique analysée, avant son exploitation.

Parmi les élevages concernés, il n'a pas été constaté de substitution de l'ensemble des animaux de race locale par des animaux croisés, mais au contraire le maintien à un niveau stable d'une population de race locale. Cela, loin d'être interprété comme une attitude conservatrice, pourrait signifier une stratégie de gestion du risque conserver une base de croisement et assurer la sécurité de l'élevage en cas de problèmes avec les croisés. C'est ce que suggère la proportion de femelles parmi ces animaux (83 p. 100), alors qu'elle était de 75 p. 100 pour l'ensemble des caprins suivis à Ngozi.

La population croisée a néanmoins varié au cours du temps. Le maintien d'un pourcentage stable à 75 p. 100 de sang Alpine (R1) pourrait indiquer une préférence relative des éleveurs pour ce niveau de croisement, correspondant à une évaluation subjective de leur adaptation au système de production. Le faible accroissement du nombre d'animaux de niveaux de croisement supérieurs (R2 et R3) serait donc lié aux difficultés de leur élevage (mortalité, sensibilité aux maladies, etc.), et à leur exploitation moins rentable sinon déficitaire en raison d'un risque pressenti par les éleveurs. La comparaison du niveau productif de ces génotypes devrait apporter des éléments de réponse. Des différences ont été percues entre les types d'éleveurs quant aux pratiques de vente des mâles et des femelles.

L'âge moyen à la vente des mâles a été comparable pour tous les éleveurs. L'objectif essentiel (répondre à des besoins monétaires) s'est traduit par la vente des chevreaux dès que ces derniers atteignaient un poids commercialisable. La tendance générale à vendre d'abord les chevreaux présentant la meilleure croissance, a entraîné le risque de saillies par des chevreaux peu performants en terme de croissance corporelle, puisqu'il n'y a que rarement castration. Ce dernier thème de vulgarisation a eu en effet peu d'impact puisqu'on n'a compté sur ce suivi que 26 castrations de chevreaux de race locale, dont 22 dans des élevages traditionnels et 58 de chevreaux croisés sur la même période.

Cependant, et contredisant l'argument de sélection négative parfois avancé (2), il semble que les meilleurs chevreaux de race locale aient été identifiés et conservés, sans doute pour la reproduction, contrairement aux chevreaux croisés.

La participation au projet a aussi modifié les conditions de cession des jeunes femelles ; celles-ci, indépendamment de leur génotype, ont été plus rapidement vendues par les éleveurs traditionnels que par les éleveurs encadrés et selectionneurs. Cela pourrait être lié à une flexibilité financière plus grande résultant du commerce du lait chez ces derniers.

Malgré ces différences de gestion, les performances d'exploitation par ventes, autoconsommation et dons des élevages ayant des caprins croisés, ont été inférieures à cellcs des élevages traditionnels. Ces derniers ont maintenu un effectif par élevage constant alors que celui des premiers est en légère diminution. Les auteurs pensent que la différence d'exploitation est due aux taux de mortalité. BA et al. (1) ont montré par simulation la variation existant entre les différents génotypes du quotient de mortalité des chevreaux de moins de six mois : 37 p.100 pour les croisés contre 23 p. 100 pour les chevreaux de race locale. La mortalité des génotypes introduits constitue ainsi une contrainte majeure pour les éleveurs ayant adopté cette innovation.

Chez ces éleveurs, l'utilisation du lait a pu compenser le moindre taux d'exploitation pour la viande. Le résultat des mesures de la production laitière illustre l'acceptation de la pratique de la traite par un tiers des éleveurs d'animaux croisés. Les producteurs choisis comme sélectionneurs ont été plus sensibles à ce thème de vulgarisation.

Cependant, malgré les efforts pour ne pas forcer les éleveurs à traire en raison de la visite de l'enquêteur, les données mesurées ne sont probablement qu'un reflet partiel de la réalité des quantités et des fréquences de traite.

\section{CONCLUSION}

Dans l'évolution des systèmes agraires des régions de haute altitude en Afrique de l'Est, les caprins se substituent progressivement aux bovins pour la production de viande et même de fumier. Le projet de Ngozi, qui vise à leur faire aussi jouer ce rôle pour la production laitière, a modifié la structure du troupeau caprin de cette province du Burundi, avec l'adoption de caprins croisés par le paysannat. Mais la modification des pratiques d'élevage la plus notable a été l'acceptation de la traite par une frac-' tion des éleveurs. Les pratiques de vente ont également changé, en particulier celle des femelles qui est devenue plus tardive. L'adoption du croisement pour disposer de chevreaux plus lourds à la vente paraît néanmoins un objectif pour un grand nombre d'éleveurs qui ne traient pas.

Les performances numériques d'exploitation ont cependant été inférieures chez ces derniers, en raison d'une plus forte mortalité des sujets croisés. Ceci est à relier à leur mode de gestion des génotypes, et donc du risque, avec un apparent refus d'une substitution complète du cheptel local par des animaux croisés, même chez les sélectionneurs les plus engagés dans le projet.

Dans ces systèmes de production économiquement fragiles, les stratégies de vente semblent essentiellement guidées par les contraintes financières. Les caprins y jouent donc un rôle de réservoir monétaire important. II en résulte de fortes fluctuations de leur population avec le risque d'abandon de l'élevage, ce qui peut ruiner le volet amélioration génétique. Le projet de développement ne doit pas négliger les dangers de cette attitude. 


\section{BIBLIOGRAPHIE}

1. BA (M.), REY (B.), MESFIN SHIBRE. Mortalité de cohortes de chevreaux croisés Alpine au Burundi. In : 2e Conférence biennale du réseau africain de recherche sur les petits ruminants, Arusha, Tanzanie, 7-11 décembre 1992

2. BRANCKAERT (R), HABONIMANA (A. de G.), NIVYOBIZI (A.). Contraintes essentielles et recherche prioritaires dans le développement de l'élevage des petits ruminants dans les zones densément peuplées des hautes terres africaines. In : Compte rendu du séminaire sur la coordination de la recherche pour le développement des petits ruminants en Afrique, CTA/CEF/GT7/IFMVT, Montpellier, 13-17 octobre, 1986. p. 71-90.

3. FAUGÈrE (O.), DOCKES (A.C.), PERROT (C.), FAUGÈrE (B.). L'élevage traditionnel des petits ruminants au Sénégal. I. Pratiques de conduite et d'exploitation des animaux chez les éleveurs de la région de Kolda. Revue Élev. Med. vét. Pays trop., 1990, 43 (2) : 249-259.

REY (B.), JACOB (U.). Goat offtake strategies in Ngozi province, Burundi. Revue Elev. Méd. vét. Pays trop., 1993, 46 (4) : 637-644

The offtake practices among goat producers in the Ngozi Province, Burundi, are described. Data were collected by monitoring 251 farmers' flocks during two years. Out of the 251 farmers, 158 maintained crossbred Alpine goats and benefited from the Ngozi Goat Production Project assistance. Variations during the two years in herd size and genetic composition on the one hand and in sales, milking practices and direct consumption by the householder on the other hand have been analysed ; variations in the strategies used by the different types of goat owners are also highlighted. Goat plays a savings role in this farming system and this was responsible for substantial variations in goat numbers within herds and for clianges in the goat owners population, with $6.3 \%$ abandons every year. The smaller the herd, the greater the risk for the goat owner to quit goat production, irrespective of the possession of crossbreds or not. Adoption of crossbreds by part of the producers had triggered some changes in the offtake practices: $65 \%$ of them took up the practice of milking and milk sales; they sold their goats at an elder mean age, although male kids were all sold at the same age. It seemed however that the best kids from the local breed were being kept for breeding, unlike crossbred kids. However, the offtake figures were lower for producers with crossbreds, who had elected to maintain a constant fraction of goats from the local breed in their herds.

Key words : Goat - Alpine goat - Farming system - Crossbreeding - Animal breeding - Milk yield - Commercialization - Livestock economics Burundi.
4. FAUGERE (O.), DOCKES (A.C.), PERROT (C.), FAUGËE (B.). L'élevage traditionnel des petits ruminants au Sénégal. II. Pratiques de conduite et d'exploitation des animaux chez les éleveurs de la région de Louga. Revue Elev. Med. vét. Pays trop., 1990, 43 (2): 261-273.

5. JONES (W. I.), EGLI (R.). Farming systems in Africa. Washington DC, World Bank, 1984. 117 p. (World Bank technical paper $n^{\circ} 27$ )

6. REY (B) JACOB (U). Farm structure and participation in the Ngozi crossbreeding project in Burundi. In : Proceedings of the 9th workshop of the SR-CRSP , Nairobi, Kenya, 3-8 march 1991. p. 141-148.

7. SCHMIDT (U). Evolution et résultats de neuf années de projet germanoburundais dans le cadre de la coopération technique. Capricorne, 1990, 3 (1) : 15-20.

8. Service National des Etudes et Statistiques (SNES). Systèmes de production et analyse de la situation alimentaire et nutritionnelle dans la région de Buyenzi. Bujumbura, Ministère du Plan, République du Burundi, 1987. 1. Rapport de synthèse, 57 p. ; 2. Les enquêtes et annexes.

REY (B.), JACOB (U.).Prácticas de explotación de caprinos én la provincia de Ngozi (Burundi). Revue Elev. Méd. vét. Pays trop., 1993, 46 (4) : $637-644$

EI presente estudio describe las prâcticas de explotación de caprinos en la Provincia de Ngozi, Burundi. Los datos fueron colectados durante dos años, mediante encuestas zootécnicas efectuadas regularmente en 251 fincas. Ciento cincuenta y ocho de los establecimientos poseían caprinos cruzados con la raza Alpina y asesoría técnica por parte del proyecto Ngozi. Durante estos dos años, se analizó la evolución del tamaño y de la composición genética del rebaño, así como las características de la venta, del consumo familiar y del ordeño de los caprinos. Se subrayan las diferencias de estrategia de manejo entre los diferentes tipos de ganaderos. En este sistema agrario, los caprinos representan un ahorro, lo que provoca una fuerte fluctuación de la población animal y la reconversión de los ganaderos, 6,3 p. 100 de los cuales abandonan la actividad caprina cada año. Este abandono de la ganadería caprina es más frecuente en los rebaños de pocas cabezas, pero es independiente del hecho de poseer caprinos cruzados. La adopción de caprinos cruzados por una parte de los criadores se acompaña de modificaciones en las prácticas de explotación : 65 p 100 de los productores aceptaron el ordeño y la venta de la leche ; las cabras se vendieron a una edad promedio mayor, mientras que los cabritos se vendieron a la misma edad. A diferencia de los jóvenes cruzados, los mejores cabritos de la raza local parecen ser el objeto de una selección. Los productores de caprinos cruzados obtuvieron rendimientos menores y mantuvieron en sus hatos una fracción constante de caprinos locales.

Palabras claves: Caprino - Cabra Alpino - Sistema ganadero - Cruzamiento - Mejoramento animal - Producción lechera - Commercialización - Economía de la cría - Burundi. 